\title{
Effects of Pitavastatin in Japanese Patients With Chronic Heart Failure - The Pitavastatin Heart Failure Study (PEARL Study) -
}

Hiroyuki Takano, MD; Hiroshi Mizuma, MD; Yoichi Kuwabara, MD; Yasunori Sato, PhD; Satoshi Shindo, MD; Norihiko Kotooka, MD; Daisuke Fujimatsu, MD; Yoshio Kobayashi, MD; Teruo Inoue, MD; Koichi Node, MD; Issei Komuro, MD on behalf of the PEARL Study Investigators

\begin{abstract}
Background: Recent clinical trials using rosuvastatin, a hydrophilic statin, did not show beneficial effects on cardiovascular events in patients with heart failure. We examined the cardioprotective effects of pitavastatin, a lipophilic statin, on Japanese patients with chronic heart failure (CHF).
\end{abstract}

\begin{abstract}
Methods and Results: A total of 574 Japanese patients with CHF were randomly assigned to the pitavastatin group $(n=288)$ or the control group $(n=286)$. There was no significant difference between the 2 groups for the primary outcome, which was a composite of cardiac death and hospitalization for worsening HF (adjusted hazard ratio (aHR): $0.922,95 \%$ confidence interval $(\mathrm{Cl}): 0.632-1.345, \mathrm{P}=0.672)$. A strongly significant statistical interaction between the effect of pitavastatin and left ventricular ejection fraction (LVEF) was found ( $P=0.004)$. In patients with $L V E F \geq 30 \%$, a significant reduction in the primary outcome (aHR: $0.525,95 \% \mathrm{Cl}: 0.308-0.896, \mathrm{P}=0.018$ ) was observed in the pitavastatin group. Pitavastatin did not show any effects on the primary outcome (aHR: 1.582, 95\% Cl: 0.890-2.813, $\mathrm{P}=0.118$ ) in the subgroup of patients with LVEF $<30 \%$.
\end{abstract}

Conclusions: Pitavastatin did not reduce cardiac death or hospitalization for worsening HF in Japanese patients with CHF. (UMIN-ID: UMINC000000428). (Circ J 2013; 77: 917-925)

Key Words: Heart failure; Hypercholesterolemia; Pitavastatin; Randomized controlled trial

$\mathbf{T}$ he number of patients with heart failure (HF) has been increasing and it is becoming a major public health problem. Although there has been considerable progress in the treatment of chronic HF (CHF) with the advent of angiotensin-converting enzyme inhibitors, angiotensin II receptor blockers, $\beta$-blockers, and aldosterone antagonists, the number of deaths from HF has been increasing steadily and further strategies are needed.

\section{Editorial p 895}

The 3-hydroxy-3-methylglutaryl coenzyme A (HMG-CoA) reductase inhibitors (ie, statins) are the most widely used agents for the treatment of hypercholesterolemia. Clinical trials have shown that treatment with statins significantly reduces the incidence of cardiovascular events in patients with coronary artery disease (CAD). ${ }^{1-3}$ In addition to their lipid-lowering action, statins have also been reported to have various pleiotropic effects, such as antiinflammatory effects, antioxidant effects, angiogenic effects, protective effects on endothelial cells, and inhibitory effects on neurohormonal activation. ${ }^{4}$ It has been demonstrated that statins inhibit the progression of HF in animal models of nonischemic HF. ${ }^{5,6}$ These effects of statins suggest a potential to ameliorate components of the

Received August 22, 2012; revised manuscript received January 29, 2013; accepted January 30, 2013; released online March 15, 2013 Time for primary review: 20 days

Department of Molecular Cardiovascular Pharmacology, Graduate School of Pharmaceutical Sciences, Chiba University, Chiba (H.T.); Department of Cardiovascular Medicine, Chiba University Graduate School of Medicine, Chiba (H.M., Y. Kuwabara, S.S., Y. Kobayashi); Clinical Research Center, Chiba University Hospital, Chiba (Y.S.); Department of Cardiovascular Medicine, Saga University, Saga (N.K., D.F., K.N.); Department of Cardiovascular Medicine, Dokkyo Medical University, Mibu (T.I.); Department of Cardiovascular Medicine, The University of Tokyo Graduate School of Medicine, Tokyo (I.K.); and Department of Cardiovascular Medicine, Osaka University Graduate School of Medicine, Osaka (I.K.), Japan

This paper was presented at the $7^{\text {th }}$ Annual Scientific Meeting of the Japanese Circulation Society, Late Breaking Clinical Trials $2-2$ (March 17, 2013, Yokohama, Japan).

Names of grants: Japan Heart Foundation and Health Labor Sciences Research Grant.

Mailing address: Issei Komuro, MD, Department of Cardiovascular Medicine, The University of Tokyo Graduate School of Medicine,

7-3-1 Hongo, Bunkyo-ku, Tokyo 113-8655, Japan. E-mail: komuro-tky@umin.ac.jp

ISSN-1346-9843 doi:10.1253/circj.CJ-12-1062

All rights are reserved to the Japanese Circulation Society. For permissions, please e-mail: cj@j-circ.or.jp 


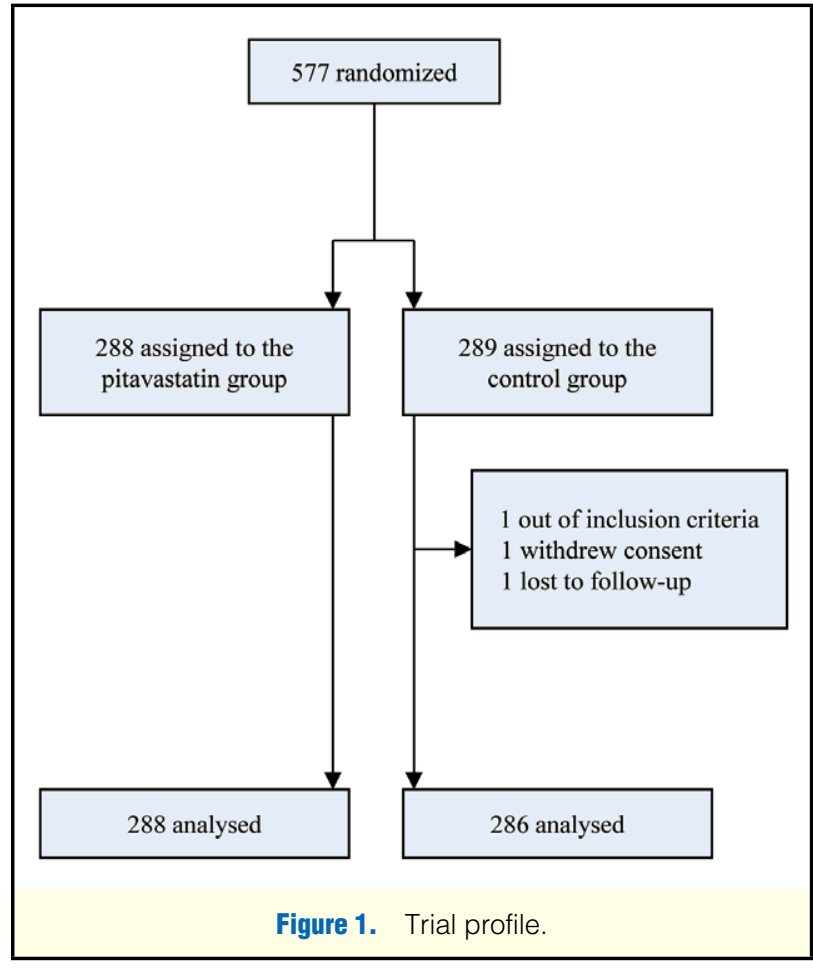

complex pathophysiology of $\mathrm{HF}$ and a promising treatment for $\mathrm{CHF}$ in the future.

Many observational studies and retrospective analyses have suggested that treatment with statins decreases the incidence of $\mathrm{HF}$ in patients with $\mathrm{CAD}$ and reduces the mortality of patients with CHF. ${ }^{7-9}$ Prospective trials have also confirmed the beneficial effects of statins on HF. ${ }^{10-12}$ On the other hand, 2 recent well-controlled randomized clinical trials have shown that statin treatment has no effect on the clinical outcomes of patients with CHF. ${ }^{13,14}$ So, it remains unclear whether treatment of patients with CHF with statins will show the same results in different studies in relation to the type of statin used, characteristics of the patients, severity of HF, and the endpoints examined.

Pitavastatin is a lipophilic statin and has longer-acting effects on decreasing low-density lipoprotein (LDL) cholesterol and increasing high-density lipoprotein (HDL) cholesterol. Pitavastatin also has high bioavailability and is minimally metabolized by the cytochrome P450 system. ${ }^{15,16}$ The Pitavastatin Heart Failure (PEARL) study was designed to evaluate the beneficial effects of pitavastatin on the incidence of cardiac death and hospitalization for worsening HF in Japanese patients with CHF. ${ }^{17}$

\section{Methods}

\section{Study Design and Patients}

The rationale and design of the PEARL study (UMIN-ID: UMINC000000428) have been previously described in detail. ${ }^{17}$ It was a multicenter, prospective, randomized, open-label, blinded-endpoint (PROBE) trial carried out in 116 Japanese institutes. The trial was approved by each center's ethics committee and all patients provided written informed consent. Eligibility criteria for enrollment were: age 20-79 years; New York Heart Association (NYHA) functional class II or III; left ventricular ejection fraction (LVEF) as measured by echocar- diography $\leq 45 \%$; stable NYHA class for 2 or more weeks prior to study randomization; mild hypercholesterolemia (serum total cholesterol level $\leq 250 \mathrm{mg} / \mathrm{dl}$ and/or serum LDL-cholesterol level $\leq 170 \mathrm{mg} / \mathrm{dl}$ ); written informed consent of the patient. The exclusion criteria included: receiving treatment with a statin prior to randomization; history of acute myocardial infarction within 3 months prior to the randomization; percutaneous coronary intervention (PCI), coronary artery bypass grafting, or cardiac resynchronization therapy-pacemaker or -defibrillator implantation performed within 3 months prior to the randomization; malignancy; serious renal or hepatic dysfunction; collagen disease; pregnancy or possible pregnancy; and lack of informed consent.

The eligible patients were randomly assigned to either the pitavastatin group ( $2 \mathrm{mg}$ daily) or the control group (no statin) at the PEARL data center using a minimization method ${ }^{18}$ with biased-coin assignment balancing on age $(<50$ years or $\geq 50$ years), sex, serum total cholesterol level $(<230 \mathrm{mg} / \mathrm{dl}$ or $\geq 230 \mathrm{mg} / \mathrm{dl}$ ), $\mathrm{LVEF}$ ( $<30 \%$ or $\geq 30 \%$ ), history of ischemic heart disease, and history of hospitalization for HF. If any patients in the control group needed further reduction of the serum total cholesterol level, lipid-lowering agents other than statins could be administered.

\section{Study Outcomes}

The primary outcome was a composite of cardiac death and hospitalization for worsening HF (hospitalization and clinical symptoms including dyspnea, shortness of breath, and peripheral edema, together with LV dysfunction by echocardiography according to AHA/ACC guidelines). For all patients, the first of these events was noted as the primary outcome. The secondary outcomes were all-cause death, cardiac death, hospitalization for worsening HF, myocardial infarction (hospitalization, chest pain, ECG-changes, and biomarkers of myocardial infarction), unstable angina (hospitalization and diagnosed by both ECG-changes corresponding with chest symptoms and coronary angiography showing $>75 \%$ stenosis according to AHA/ACC guidelines), stroke (hospitalization and diagnosed by CT and/or MRI), PCI, and surgical therapy for worsening HF. Death was classified as cardiac unless a definite non-cardiovascular reason was identified. All outcomes recorded in this study were adjudicated blindly by an independent endpoint committee (Appendix S1) on the basis of prespecified definitions and procedures.

\section{Statistical Analysis}

The sample size calculation has been previously described in detail. ${ }^{17}$ Our trial required 470 patients with a primary outcome occurring within 48 months to achieve $80 \%$ power to detect $43 \%$ relative reduction in the risk of the primary outcome in the pitavastatin group as compared with the control group (with a 2 -sided $\alpha$ of 0.05 ) on the condition of an expected mortality rate at 24 months of $20 \%$ in the control group. Efficacy was evaluated in 2 interim analyses performed by an independent data and safety monitoring committee at the last patient registration and 1 year after the completion of enrollment. Significance was evaluated using the method of Lan and DeMets and the O'Brien-Fleming boundary (the stopping boundary, $\mathrm{P} \leq 0.000011$ at the first interim analysis, $\mathrm{P} \leq 0.0089$ at the second interim analysis, and $\mathrm{P} \leq 0.041$ at the final analysis).

Comparability of baseline characteristics between the 2 groups was assessed by 2-sample t-test with equal variance or the Mann-Whitney U test for continuous variables, and Fisher's exact test for categorical variables. The analyses of the adju- 


\begin{tabular}{|c|c|c|c|}
\hline & Control $(n=286)$ & Pitavastatin (n=288) & P-value \\
\hline \multicolumn{4}{|l|}{ Age } \\
\hline Age (years) & $62.2 \pm 12.0$ & $62.9 \pm 11.7$ & 0.47 \\
\hline Age $\geq 50$ years, $n(\%)$ & $246(86.0)$ & $249(86.5)$ & 0.87 \\
\hline Female, n (\%) & $51(17.8)$ & $55(19.1)$ & 0.69 \\
\hline NYHA functional class, $n$ (\%) & & & 0.50 \\
\hline II & $259(90.6)$ & $256(88.9)$ & \\
\hline III & $27(9.4)$ & $32(11.1)$ & \\
\hline LVEF (\%) & $34.0 \pm 7.7$ & $33.9 \pm 8.9$ & 0.88 \\
\hline LVEF $\geq 30 \%$, no. (\%) & $206(72.0)$ & 207 (71.9) & 0.96 \\
\hline \multicolumn{4}{|l|}{ Blood pressure (mmHg) } \\
\hline Systolic & $118.4 \pm 17.7$ & $120.3 \pm 18.0$ & 0.20 \\
\hline Diastolic & $71.3 \pm 11.2$ & $72.4 \pm 11.0$ & 0.23 \\
\hline Heart rate (beats/min) & $72.6 \pm 11.4$ & $73.2 \pm 13.0$ & 0.64 \\
\hline HF cause, $n(\%)$ & & & 0.80 \\
\hline IHD & $80(28.0)$ & $77(26.7)$ & \\
\hline $\mathrm{DCM}$ & $149(52.1)$ & $161(55.9)$ & \\
\hline Hypertensive heart disease & $11(3.8)$ & $10(3.5)$ & \\
\hline Valvular disease & $12(4.2)$ & $15(5.2)$ & \\
\hline Others & $34(11.9)$ & $25(8.7)$ & \\
\hline \multicolumn{4}{|l|}{ Medical treatment, $\mathbf{n}(\%)$} \\
\hline Digitalis & $72(25.2)$ & $71(24.7)$ & 0.86 \\
\hline$\beta$-blockers & 204 (71.3) & $212(73.6)$ & 0.58 \\
\hline Diuretic drugs & $218(76.2)$ & 211 (73.3) & 0.37 \\
\hline ACE inhibitors or ARBs & $235(82.2)$ & $247(85.8)$ & 0.21 \\
\hline Calcium channel blockers & $49(17.1)$ & $42(14.6)$ & 0.39 \\
\hline Anticoagulants & $132(46.2)$ & $117(40.6)$ & 0.18 \\
\hline Antiplatelet agent & $128(44.8)$ & $121(42.0)$ & 0.43 \\
\hline \multicolumn{4}{|l|}{ Medical history, n (\%) } \\
\hline Hospitalization for HF & & & 0.90 \\
\hline At least 2 months before & $131(45.8)$ & $127(44.1)$ & \\
\hline Within 2 months & $47(16.4)$ & $50(17.4)$ & \\
\hline MI & $73(25.5)$ & $71(24.7)$ & 0.80 \\
\hline $\mathrm{CABG}$ or $\mathrm{PCl}$ & $56(19.6)$ & $60(20.8)$ & 0.70 \\
\hline Hypertension & $129(45.1)$ & $131(45.5)$ & 0.92 \\
\hline Diabetes mellitus & $79(27.6)$ & $78(27.1)$ & 0.88 \\
\hline Stroke & $24(8.4)$ & $15(5.2)$ & 0.12 \\
\hline \multicolumn{4}{|l|}{ Laboratory measurements } \\
\hline Total cholesterol (mg/dl) & $201.2 \pm 32.6$ & $203.2 \pm 32.2$ & 0.45 \\
\hline LDL-cholesterol (mg/dl) & $125.5 \pm 32.7$ & $125.2 \pm 28.1$ & 0.93 \\
\hline HDL-cholesterol (mg/dl) & $50.8 \pm 15.1$ & $50.7 \pm 14.5$ & 0.92 \\
\hline \multicolumn{4}{|l|}{$\mathrm{hsCRP}(\mathrm{ng} / \mathrm{ml})$} \\
\hline Median & 839.0 & 834.0 & 0.85 \\
\hline Interquartile range & $394.0-2,300.0$ & $391.0-2,535.0$ & \\
\hline
\end{tabular}

These data are the number of patients (\%) or mean (SD).

NYHA, New York Heart Association; LVEF, left ventricular ejection fraction; HF, heart failure; IHD, ischemic heart disease; DCM, dilated cardiomyopathy; ACE, angiotensin-converting enzyme; ARBs, angiotensin II receptor blockers; $\mathrm{MI}$, myocardial infarction; CABG, coronary artery bypass grafting; PCl, percutaneous coronary intervention; LDL, low-density lipoprotein; HDL, high-density lipoprotein; hsCRP, high-sensitivity C-reactive protein.

dicated primary and secondary outcomes were conducted on data from all patients who had undergone randomization, according to the intention-to-treat principle, with the use of Kaplan-Meier estimates and Cox proportional-hazards models. Hazard ratio, 95\% confidence interval $(\mathrm{CI})$, and $\mathrm{P}$ value were calculated with the use of models adjusted for the following prespecified baseline prognostic factors: age, sex, serum total cholesterol level, LVEF, history of ischemic heart dis- ease, and history of hospitalization for HF. Sensitivity analyses were also performed by means of unadjusted Cox models.

The consistency of the treatment effect was assessed among 19 prespecified subgroups. The effect in each subgroup was analyzed with a Cox proportional-hazards model without adjustment for covariates. The interaction between treatment and subgroup was evaluated by Cox proportional-hazards model with terms for treatment, subgroup, and their interaction. 


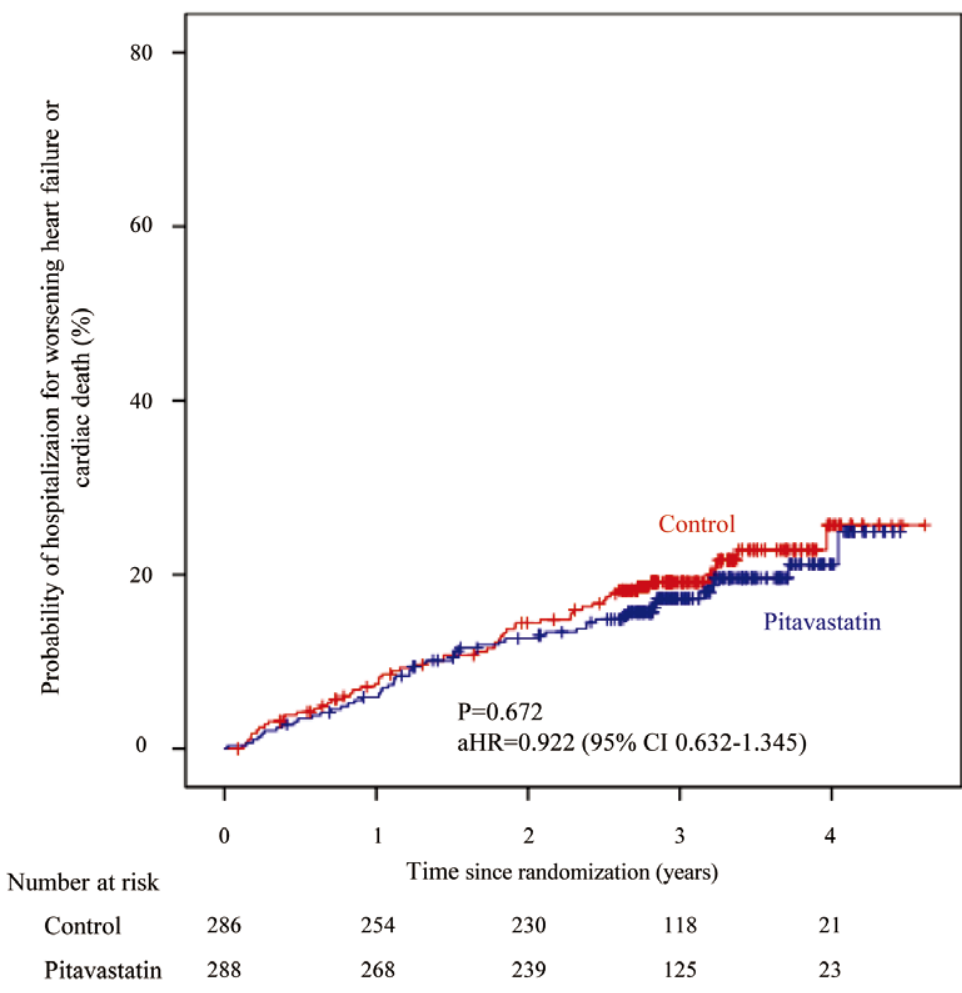

Figure 2. Kaplan-Meier curve for the primary outcome. The P-value was calculated by logrank test. aHR, adjusted hazard ratio.

All comparisons were planned and the tests were 2-sided. $\mathrm{P}<0.05$ was considered to indicate a statistically significant difference. All statistical analyses were conducted using SAS software program, version 9.2 (SAS Institute Inc, Cary, NC, USA).

\section{Study Management}

Data on the primary and secondary outcomes and adverse events were collected at various time points and interim analyses were performed every year after the initiation of the study to evaluate both the events and treatment safety. An independent endpoint committee (Appendix S1) consisting of 3 members, who were blinded to any information relating to the group allocations, evaluated each event and classified the results. An independent data and safety monitoring board (Appendix S2) comprised 3 members who reviewed all reports from the endpoint committee to advise early termination of the study for safety, scientific or ethical reasons. A steering committee (Appendix S3) was responsible for the study design and scientific execution of the study.

The independent data and safety monitoring board's charter specified interim analyses of the primary outcome at the last patient registration and 1 year after the last registration, with a statistical stopping guideline for an overwhelming benefit.

\section{Results}

We enrolled 577 symptomatic CHF patients with mild hypercholesterolemia from June 2006 to June 2008. All the patients were followed up until April 2011 for primary and secondary outcomes. Finally, 577 patients were randomly assigned to the 2 groups (Figure 1). Of them, 288 were assigned to the pitavastatin group and 289 to the control group. As shown in
Figure 1, 1 patient was excluded, 1 patient withdrew consent after random allocation, and 1 patient was lost to follow-up. We obtained complete endpoint information at the end of the study for 574 patients. The median duration of follow-up was 35.5 months. Table 1 shows the baseline characteristics of the patients enrolled; there were no significant differences between the 2 groups. The patients had a mean age of 62.6 years and $86.2 \%$ of them were older than 50 years. The mean LVEF was $34.0 \%$ and $89.7 \%$ of the patients were classified as NYHA class II. The cause of HF was ischemic heart disease in 157 $(27.4 \%)$ patients and dilated cardiomyopathy in $310(54.0 \%)$ patients.

The primary outcome occurred in $52(18.0 \%)$ patients in the pitavastatin group and 57 (19.9\%) patients in the control group. There was no significant difference in the primary outcome between the 2 groups (adjusted hazard ratio (aHR): 0.922, 95\% CI: 0.632-1.345, $\mathrm{P}=0.672$ ) (Figure 2). In the secondary outcomes, there were no significant differences between the 2 groups (Table 2). The number of non-cardiac death was smaller in the pitavastatin group $(n=3)$ than in the control group $(n=15)$. In the predefined subgroup analysis (Figure 3), we found a strongly significant statistical interaction between the effect of pitavastatin and LVEF $(\mathrm{P}=0.004)$. In the subgroup of patients with $L V E F \geq 30 \%(n=413)$, pitavastatin was associated significantly fewer occurrences of the primary outcome (aHR: 0.525 , 95\% CI: 0.308-0.896, $\mathrm{P}=0.018$ ) (Figure 4A). On the other hand, pitavastatin did not show any effects for the primary outcome in the subgroup of patients with LVEF $<30 \%$ (aHR: 1.582 , 95\% CI: 0.890-2.813, $\mathrm{P}=0.118$ ) (Figure 4B). As for the secondary outcomes, pitavastatin significantly reduced all-cause death and hospitalization due to worsening HF in the subgroup of patients with LVEF $\geq 30 \%$ (Table 3 ).

In the pitavastatin group, serum level of LDL-cholesterol 


\begin{tabular}{|c|c|c|c|c|}
\hline & $\begin{array}{l}\text { Control } \\
(n=286)\end{array}$ & $\begin{array}{l}\text { Pitavastatin } \\
(\mathrm{n}=\mathbf{2 8 8})\end{array}$ & *aHR (95\% Cl) & $P$ value \\
\hline \multicolumn{5}{|l|}{ Primary } \\
\hline $\begin{array}{l}\text { Composite of cardiac death and hospitalization } \\
\text { due to worsening HF }\end{array}$ & $57(19.9 \%)$ & $52(18.0 \%)$ & $0.922(0.632-1.345)$ & 0.672 \\
\hline \multicolumn{5}{|l|}{ Secondary } \\
\hline All-cause death & $37(12.9 \%)$ & $27(9.37 \%)$ & $0.727(0.441-1.198)$ & 0.211 \\
\hline Cardiac death & $22(7.7 \%)$ & $24(8.3 \%)$ & $1.097(0.611-1.969)$ & 0.756 \\
\hline Hospitalization due to worsening HF & $47(16.4 \%)$ & $39(13.5 \%)$ & $0.836(0.547-1.282)$ & 0.411 \\
\hline Ml or unstable angina & $8(2.8 \%)$ & $3(1.0 \%)$ & $0.396(0.103-1.519)$ & 0.177 \\
\hline Stroke & $9(3.2 \%)$ & $8(2.8 \%)$ & $0.886(0.340-2.306)$ & 0.804 \\
\hline $\mathrm{PCl}$ & $6(2.1 \%)$ & $3(1.0 \%)$ & $0.374(0.083-1.687)$ & 0.200 \\
\hline Surgical therapy for HF & $11(3.8 \%)$ & $12(4.2 \%)$ & $1.043(0.458-2.374)$ & 0.920 \\
\hline
\end{tabular}

${ }^{*}$ Adjusted for the primary outcome in age, sex, total cholesterol, LVEF, IHD, and history of hospitalization for HF.

Data are the numbers of patients (\%).

aHR, adjusted hazard ratio; $\mathrm{Cl}$, confidence interval. Other abbreviations as in Table 1.

significantly decreased from $125.3 \mathrm{mg} / \mathrm{dl}$ at baseline to $83.6 \mathrm{mg} / \mathrm{dl}$ after 2 years $(-32.5 \%$ [ -29.4 to -35.6$]), \mathrm{P}<0.0001$, paired t-test $)$. The serum level of LDL-cholesterol also significantly decreased from $125.5 \mathrm{mg} / \mathrm{dl}$ at baseline to $115.4 \mathrm{mg} / \mathrm{dl}$ after 2 years in the control group $(-7.2 \%$ [ -10.7 to -3.7$], \mathrm{P}<0001$, paired t-test). The percent change in LDL-cholesterol in the pitavastatin group was significantly high compared with the control group after 2 years $(\mathrm{P}<0.001$, Mann-Whitney $\mathrm{U}$ test $)$. In the pitavastatin group, the serum level of HDL-cholesterol significantly increased from $50.7 \mathrm{mg} / \mathrm{dl}$ at baseline to $52.1 \mathrm{mg} / \mathrm{dl}$ after 2 years (5.1\% [2.1 to 8.1], $\mathrm{P}=0.0009$, paired t-test). In the control group, the serum level of HDL-cholesterol did not significantly change $(50.8 \mathrm{mg} / \mathrm{dl}$ at baseline to $50.2 \mathrm{mg} / \mathrm{dl}$ after 2 years $(0.5 \%[-2.5$ to 3.6$], \mathrm{P}=0.73$, paired t-test)). The percent change in HDLcholesterol in the pitavastatin group was significantly high compared with the control group after 2 years $(\mathrm{P}=0.023$, MannWhitney $\mathrm{U}$ test). Although there was a trend for higher rates of muscle-related symptoms and elevation in level of creatine phosphokinase, we did not note any significant differences in the adverse events between the 2 groups (Table 4 ).

\section{Discussion}

The PEARL study aimed to investigate the effects of pitavastatin on the incidence of cardiac death and hospitalization for worsening HF in Japanese patients with CHF. Our findings confirm that pitavastatin does not reduce cardiac death and hospitalization for worsening HF entirely, but significantly reduces those events in the subgroup of patients with LVEF $\geq 30 \%$.

It is well known that treatment with statins significantly reduces the incidence of cardiovascular events in patients at high risk, especially those with CAD, irrespective of the baseline cholesterol levels. ${ }^{19}$ Several retrospective analyses and large observational studies have suggested that treatment with statins decreases the incidence of HF and reduces mortality in patients with $\mathrm{CHF}^{7-9}$ Furthermore, prospective trials assessing the effects of statins on surrogate endpoints such as biomarkers and echocardiographic parameters demonstrated the beneficial effects of statins on patients with CHF. ${ }^{10-12}$ Metaanalyses of statin treatment in randomized clinical trials also confirmed a reduction of cardiovascular mortality in patients with CHF of both ischemic and nonischemic etiologies. ${ }^{4}$ These results support the pleiotropic effects of statins demonstrated by basic research and the beneficial effects of statins on patients with CHF.

Recently, the results of 2 well-controlled randomized trials have been published. ${ }^{13,14}$ The CORONA (Controlled rosuvastatin multinational study in heart failure) study randomized 5,011 patients with symptomatic CHF of ischemic etiology to $10 \mathrm{mg}$ rosuvastatin or placebo. ${ }^{13}$ The GISSI (Gruppo Italiano per lo Studio della Sopravvivenza nell'Insufficienza cardiaca)HF (Heart failure) trial randomized 4,631 patients with symptomatic $\mathrm{CHF}$ of both ischemic and nonischemic etiologies to $10 \mathrm{mg}$ rosuvastatin or placebo. ${ }^{14}$ The treatment with rosuvastatin did not affect the primary endpoints in the 2 studies. ${ }^{13,14}$ It remains unknown at present whether the results of the CORONA study and the GISSI-HF trial can be applied in the different patient selection or in the different kind of statin. Interestingly, meta-analysis has reported that the effects of statins on HF are not a class effect and a significant benefit was noted using lipophilic atorvastatin but not hydrophilic rosuvastatin. ${ }^{20}$ Several studies have also reported that atorvastatin has beneficial effects on the patients with HF, but rosuvastatin does not show the beneficial effects. ${ }^{21-24}$ Therefore, there is a possibility that lipophilic statins are more useful than hydrophilic statins for the treatment of CHF.

In the PEARL study, we used pitavastatin to evaluate the beneficial effects of statin therapy on Japanese patients with CHF. Pitavastatin is a lipophilic agent, whereas rosuvastatin is a hydrophilic agent. Statins potently reduce the serum LDLcholesterol level by inhibiting the synthesis of mevalonate and inducing the expression of LDL receptors, mainly in the hepatocytes. Among statins, pitavastatin has been reported to most strongly increase LDL receptor mRNA expression. ${ }^{25}$ These data indicate that there is minimal likelihood of deleterious effects resulting from inhibition of the mevalonate pathway in the case of pitavastatin. As pitavastatin is hardly metabolized through the cytochrome P450-mediated pathway, pitavastatin is expected to have little interaction with other agents metabolized through the $\mathrm{P} 450$ pathway. ${ }^{26}$ This property may be potentially beneficial for patients with $\mathrm{CHF}$ who take several kinds of medicines for the treatment of HF.

There are some differences in the characteristics of patients and protocol among the PEARL study, the CORONA study, and the GISSI-HF trial. The ratio of patients with ischemic HF was $40 \%$ in the GISSI-HF trial and $100 \%$ in the CORONA study, while only $27.4 \%$ of patients had ischemic HF in the 


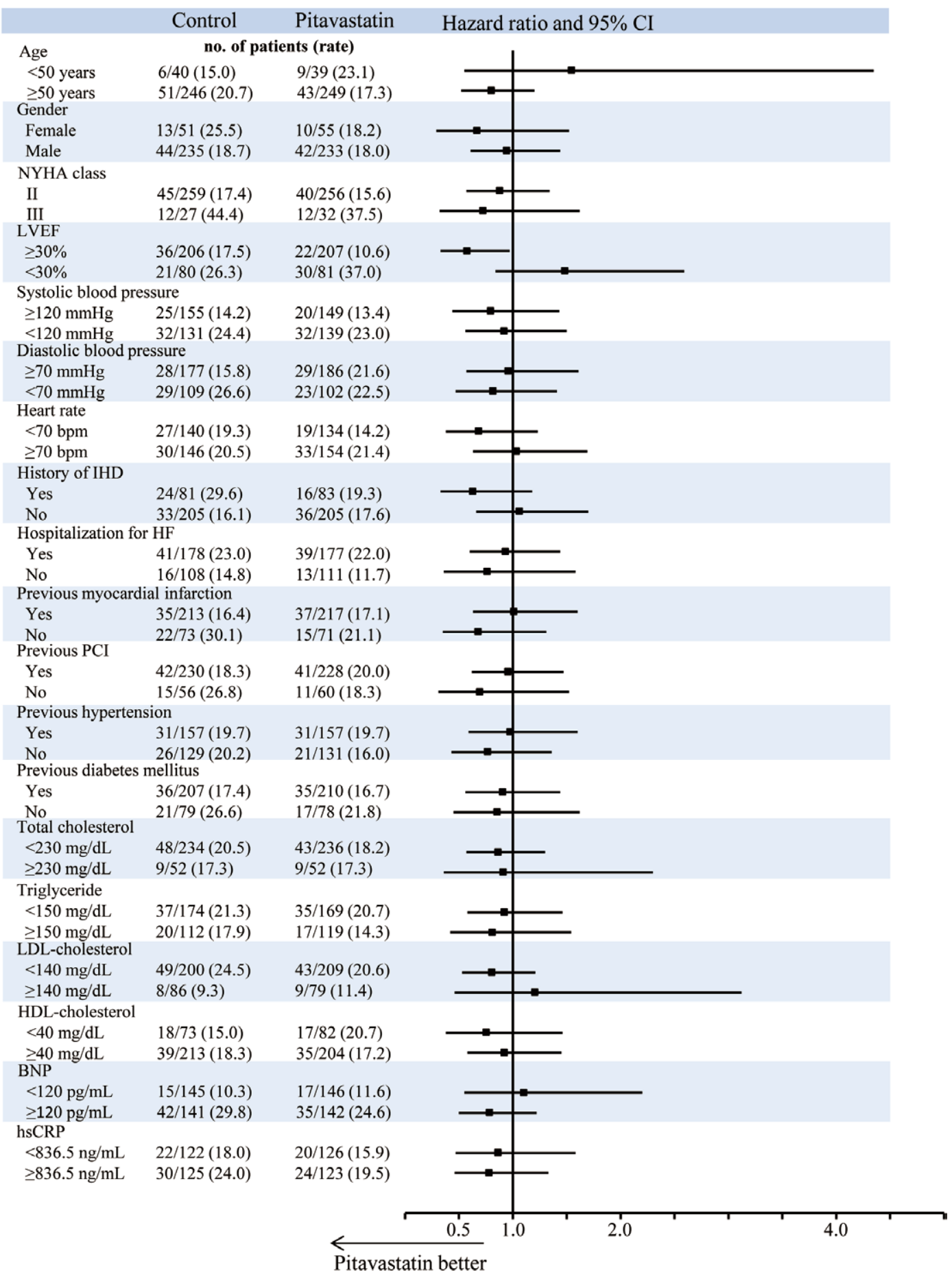

Figure 3. Predefined subgroup analysis for the primary outcome. bpm, beats/min; NYHA, New York Heart Association; LVEF, left ventricular ejection fraction; IHD, ischemic heart disease; HF, heart failure; $\mathrm{PCl}$, percutaneous coronary intervention; LDL, lowdensity lipoprotein; HDL, high-density lipoprotein; BNP, B-type natriuretic peptide; hsCRP, high-sensitivity C-reactive protein.

PEARL study. The mean age of the patients was 73, 68, and 63 years in the CORONA, the GISSI-HF, and the PEARL, respectively. The enrolled patients appear to be less symptomatic in the PEARL study (NYHA II $89.7 \%$, III 10.3\%, IV 0\%) than in the CORONA study (NYHA II $37.0 \%$, III $61.5 \%$, IV
$1.5 \%$ ) and the GISSI-HF trial (NYHA II $62.5 \%$, III $35.0 \%$, IV $2.5 \%$ ). Furthermore, all the patients enrolled in the PEARL study were Japanese. There are differences in the responses to therapeutic drugs between Asian and Western populations. ${ }^{27}$ Pharmacokinetic studies have demonstrated higher plasma 

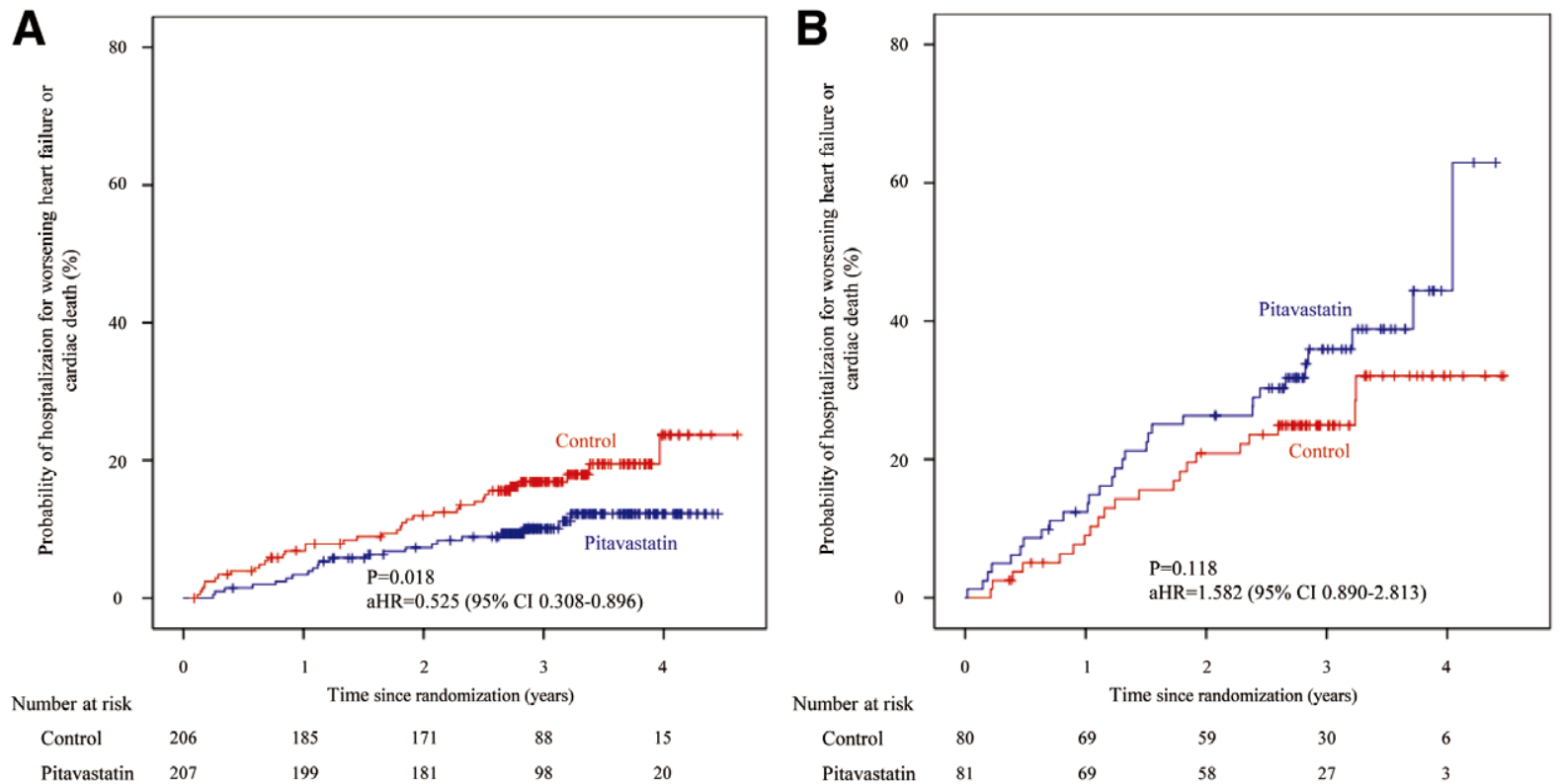

Figure 4. Kaplan-Meier curves for the primary outcome in the subgroup of patients with LVEF $\geq 30 \%$ (A) and in the subgroup of patients with LVEF $<30 \%(B)$. The P-values were calculated by log-rank test. aHR, adjusted hazard ratio; LVEF, left ventricular ejection fraction.

Table 3. Outcomes of Subgroup of Patients With LVEF $\geq 30 \%$ or $<30 \%$

Control $(n=206)$

Pitavastatin $(\mathrm{n}=\mathbf{2 0 7})$

*aHR (95\% Cl)

$P$ value

\section{LVEF $\geq 30 \%$}

Primary

Composite of cardiac death and hospitalization due to worsening HF

$36(17.5 \%)$

$22(10.7 \%)$

$0.525(0.308-0.896)$

0.018

\section{Secondary}

All-cause death

$22(10.7 \%)$
$12(5.9 \%)$
$30(14.6 \%)$
$7(3.4 \%)$
$5(2.5 \%)$
$4(2.0 \%)$
$6(3.0 \%)$

$12(5.8 \%)$

$9(4.3 \%)$

$18(8.7 \%)$

$3(1.4 \%)$

$8(3.9 \%)$

$3(1.4 \%)$

$6(2.9 \%)$

Surgical therapy for HF

Control $(n=80)$

Primary

Composite of cardiac death and hospitalization due to worsening HF

\section{Secondary}

All-cause death

Cardiac death

Hospitalization due to worsening HF

MI or unstable angina

Stroke

$\mathrm{PCl}$

Surgical therapy for HF
$21(26.3 \%)$

$15(18.7 \%)$
$10(12.5 \%)$
$17(21.2 \%)$
$1(1.2 \%)$
$4(5.0 \%)$
$2(2.5 \%)$
$5(6.3 \%)$

Pitavastatin $(n=81)$

$30(37.0 \%)$

$15(18.5 \%)$
$15(18.5 \%)$
$21(25.9 \%)$
$0(0.0 \%)$
$0(0.0 \%)$
$0(0.0 \%)$
$6(7.4 \%)$

$0.485(0.238-0.989)$

$0.695(0.290-1.666)$

$0.508(0.282-0.915)$

$0.432(0.110-1.701)$

$1.484(0.481-4.578)$

$0.406(0.074-2.223)$

$0.994(0.317-3.116)$

*aHR $(95 \% \mathrm{Cl})$

$1.582(0.890-2.813)$

0.118

$\begin{array}{cl}0.931(0.440-1.972) & 0.85 \\ 1.363(0.587-3.162) & 0.471 \\ 1.417(0.735-2.729) & 0.298 \\ \text { NA } & 0.999 \\ \text { NA } & 0.997 \\ \text { NA } & 0.999 \\ 1.019(0.293-3.544) & 0.976\end{array}$

${ }^{*}$ Adjusted for the primary outcome in age, sex, total cholesterol, IHD, and history of hospitalization for HF.

Data are the numbers of patients (\%).

NA, not available. Other abbreviations as in Tables 1,2. 


\begin{tabular}{|lcc|}
\hline Table 4. Adverse Events & Control & Pitavastatin \\
$(\mathbf{n = 2 8 6 )}$ & $(\mathbf{n = 2 8 8 )}$ \\
Myalgia or arthralgia & $1(0.35)$ & $4(1.39)$ \\
CK increase & $0(0.00)$ & $3(1.04)$ \\
Gastrointestinal disorders & $3(1.04)$ & $2(0.69)$ \\
Giddiness & $0(0.00)$ & $4(1.39)$ \\
Liver disorder & $2(0.70)$ & $2(0.69)$ \\
Renal disorder & $0(0.00)$ & $3(1.04)$ \\
Rashes & $2(0.70)$ & $1(0.35)$ \\
Anemia & $1(0.35)$ & $1(0.35)$ \\
\hline
\end{tabular}

Data are the numbers of patients (\%).

CK, creatine kinase.

levels of statins in Asians as compared with Caucasians. ${ }^{28}$ The high responses of Asians to statins are thought to be related to genetic differences in the metabolism of statins. ${ }^{28}$ The kind of statins used in the PEARL study, the CORONA study, and the GISSI-HF trial was also different.

Concerns have been raised about the possible deleterious effects of statins. Circulating lipoproteins have the ability to bind and detoxify bacterial lipopolysaccharide. Because lipopolysaccharide stimulates the release of inflammatory cytokines, statins may increase inflammation in patients with HF. ${ }^{29}$ Moreover, statins decrease the synthesis of not only cholesterol but also other downstream products in the mevalonate pathway. Coenzyme Q10 (ubiquinone), which is an essential cofactor in the mitochondrial electron transport chain, plays an important role in the mitochondrial respiratory chain and has an antioxidant function. ${ }^{30,31}$ However, a substudy of the CORONA demonstrated that plasma coenzyme Q10 concentration was not an independent predictor of clinical outcomes in the patents with HF. ${ }^{32}$ Indeed, the serious adverse effects were not recognized in the CONONA, the GISSI-HF, and the PEARL.

\section{Study Limitations}

First, as the number of the enrolled patients was small, it is possible that the follow-up period was too short to demonstrate statistical significance. We assumed that the hazard ratio of cardiac events (cardiac death and hospitalization for worsening HF) of the pitavastatin group to the control group was 0.57 as previously reported. ${ }^{17}$ However, the hazard ratio of cardiac events recognized between the 2 groups was more than 0.57 in the present study because the number of cardiac events was small. In the subgroup of patients with LVEF $\geq 30 \%$, pitavastatin showed significantly fewer cardiac events of the primary outcome because the hazard ratio was close to 0.57 . Therefore, sufficient statistical power was achieved to address the objective in the subgroup analysis. Second, we used a PROBE design but not a double-blind design in the present study. However, the PROBE design is a cost-effective alternative to the double-blind trial, and has advantages of being more similar to clinical practice and improved patient compliance without affecting the valuable blinded endpoint information. Third, the inclusion criteria of the study were NYHA class II or III, and LVEF $\leq 45 \%$, but the majority of the enrolled patients had mild HF symptoms. Therefore, the number of the primary outcome was relatively low compared with other studies (the rate of cardiac death: $8 \%$ in the PEARL, $19 \%$ in the CORONA, and $21 \%$ in the GISSI-HF; the rate of hospitalization due to worsening HF: $15 \%$ in the PEARL, $26 \%$ in the
CORONA, and $28 \%$ in the GISSI-HF). ${ }^{13,14}$ Fourth, pitavastatin showed the significant reduction in cardiac death and hospitalization for worsening $\mathrm{HF}$ in the subgroup of patients with $\mathrm{LVEF} \geq 30 \%$, but our study was not designed to investigate the effects of pitavastatin on HF patients with LVEF $\geq 30 \%$. And the pathophysiological mechanisms underlying the effects of pitavastatin on the HF patients with LVEF $\geq 30 \%$ in the present study remain largely unexplained. These findings require confirmation in a larger randomized clinical trial.

The PEARL study is the first to evaluate the effects and safety of pitavastatin in Japanese patients with CHF. Pitavastatin showed significant reduction of cardiac death and hospitalization for worsening HF in the group of patients with LVEF $\geq 30 \%$, although not in the entire population. These results suggest that pitavastatin has protective effects on Japanese patients with mild HF.

\section{Acknowledgments}

This study was supported by research grants from Japan Heart Foundation and partly supported by Health Labor Sciences Research Grant. The authors gratefully acknowledge the assistance of Ms. Naomi Harada, Mr. Hiroshi Komoda, Ms. Chiharu Kawaguchi, Ms. Sae Katafuchi, Ms. Nanae Tanemura, and Ms. Yuko Nakane.

\section{Disclosures}

None.

\section{References}

1. LaRosa JC, He J, Vupputuri S. Effect of statins on risk of coronary disease: A meta-analysis of randomized controlled trials. JAMA 1999; 282: $2340-2346$.

2. Pignone M, Phillips C, Mulrow C. Use of lipid lowering drugs for primary prevention of coronary heart disease: Meta-analysis of randomised trials. BMJ 2000; 321: 983-986.

3. Baigent C, Keech A, Kearney PM, Blackwell L, Buck G, Pollicino C, et al. Efficacy and safety of cholesterol-lowering treatment: Prospective meta-analysis of data from 90,056 participants in 14 randomised trials of statins. Lancet 2005; 366: 1267-1278.

4. Ramasubbu K, Estep J, White DL, Deswal A, Mann DL. Experimental and clinical basis for the use of statins in patients with ischemic and nonischemic cardiomyopathy. J Am Coll Cardiol 2008; 51: 415-426.

5. Zaca V, Rastogi S, Imai M, Wang M, Sharov VG, Jiang A, et al. Chronic monotherapy with rosuvastatin prevents progressive left ventricular dysfunction and remodeling in dogs with heart failure. J Am Coll Cardiol 2007; 50: 551-557.

6. Kameda Y, Hasegawa H, Kubota A, Tadokoro H, Kobayashi Y, Komuro I, et al. Effects of pitavastatin on pressure overload-induced heart failure in mice. Circ J 2012; 76: 1159-1168.

7. Shepherd J, Blauw GJ, Murphy MB, Bollen EL, Buckley BM, Cobbe SM, et al. Pravastatin in elderly individuals at risk of vascular disease (PROSPER): A randomised controlled trial. Lancet 2002; 360: 1623 1630.

8. Horwich TB, MacLellan WR, Fonarow GC. Statin therapy is associated with improved survival in ischemic and non-ischemic heart failure. J Am Coll Cardiol 2004; 43: 642-648.

9. Go AS, Lee WY, Yang J, Lo JC, Gurwitz JH. Statin therapy and risks for death and hospitalization in chronic heart failure. JAMA 2006; 296: $2105-2111$.

10. Node K, Fujita M, Kitakaze M, Hori M, Liao JK. Short-term statin therapy improves cardiac function and symptoms in patients with idiopathic dilated cardiomyopathy. Circulation 2003; 108: 839-843.

11. Wojnicz R, Wilczek K, Nowalany-Kozielska E, Szygula-Jurkiewicz B, Nowak J, Polonski L, et al. Usefulness of atorvastatin in patients with heart failure due to inflammatory dilated cardiomyopathy and elevated cholesterol levels. Am J Cardiol 2006; 97: 899-904.

12. Sola S, Mir MQ, Lerakis S, Tandon N, Khan BV. Atorvastatin improves left ventricular systolic function and serum markers of inflammation in nonischemic heart failure. J Am Coll Cardiol 2006; 47: $332-337$.

13. Kjekshus J, Apetrei E, Barrios V, Bohm M, Cleland JG, Cornel JH, et al. Rosuvastatin in older patients with systolic heart failure. $N \mathrm{Engl}$ J Med 2007; 357: 2248-2261. 
14. Tavazzi L, Maggioni AP, Marchioli R, Barlera S, Franzosi MG, Latini $\mathrm{R}$, et al. Effect of rosuvastatin in patients with chronic heart failure (the GISSI-HF trial): A randomised, double-blind, placebo-controlled trial. Lancet 2008; 372: 1231-1239.

15. Saito Y, Yamada N, Teramoto T, Itakura H, Hata Y, Nakaya N, et al. A randomized, double-blind trial comparing the efficacy and safety of pitavastatin versus pravastatin in patients with primary hypercholesterolemia. Atherosclerosis 2002; 162: $373-379$.

16. Iglesias P, Diez JJ. New drugs for the treatment of hypercholesterolaemia. Expert Opin Investig Drugs 2003; 12: 1777-1789.

17. Mizuma H, Inoue T, Takano H, Shindo S, Oka T, Fujimatsu D, et al. Rationale and design of a study to evaluate effects of pitavastatin on Japanese patients with chronic heart failure: The pitavastatin heart failure study (PEARL study). Int J Cardiol 2012; 156: 144-147.

18. Pocock SJ, Simon R. Sequential treatment assignment with balancing for prognostic factors in the controlled clinical trial. Biometrics 1975; 31: $103-115$.

19. Sever PS, Dahlof B, Poulter NR, Wedel H, Beevers G, Caulfield M, et al. Prevention of coronary and stroke events with atorvastatin in hypertensive patients who have average or lower-than-average cholesterol concentrations, in the Anglo-Scandinavian Cardiac Outcomes Trial--Lipid Lowering Arm (ASCOT-LLA): A multicentre randomised controlled trial. Lancet 2003; 361: 1149-1158.

20. Lipinski MJ, Cauthen CA, Biondi-Zoccai GG, Abbate A, Vrtovec B, Khan BV, et al. Meta-analysis of randomized controlled trials of statins versus placebo in patients with heart failure. Am J Cardiol 2009; 104: 1708-1716.

21. Krum H, Ashton E, Reid C, Kalff V, Rogers J, Amarena J, et al. Double-blind, randomized, placebo-controlled study of high-dose HMG CoA reductase inhibitor therapy on ventricular remodeling, pro-inflammatory cytokines and neurohormonal parameters in patients with chronic systolic heart failure. J Card Fail 2007; 13: 1-7.

22. Vrtovec B, Okrajsek R, Golicnik A, Ferjan M, Starc V, Schlegel TT, et al. Atorvastatin therapy may reduce the incidence of sudden cardiac death in patients with advanced chronic heart failure. J Card Fail 2008; 14: 140-144.

23. Tsutamoto T, Sakai H, Ibe K, Yamaji M, Kawahara C, Nakae I, et al. Effect of atorvastatin vs. rosuvastatin on cardiac sympathetic nerve activity in non-diabetic patients with dilated cardiomyopathy. Circ J 2011; 75: 2160-2166.
24. Ashton E, Windebank E, Skiba M, Reid C, Schneider H, Rosenfeldt F, et al. Why did high-dose rosuvastatin not improve cardiac remodeling in chronic heart failure? Mechanistic insights from the UNIVERSE study. Int J Cardiol 2011; 146: 404-407.

25. Morikawa S, Umetani M, Nakagawa S, Yamazaki H, Suganami H, Inoue K, et al. Relative induction of mRNA for HMG CoA reductase and LDL receptor by five different HMG-CoA reductase inhibitors in cultured human cells. J Atheroscler Thromb 2000; 7: 138-144.

26. Fujino H, Saito T, Tsunenari Y, Kojima J. Interaction between several medicines and statins. Arzneimittelforschung 2003; 53: 145-153.

27. Kim K, Johnson JA, Derendorf H. Differences in drug pharmacokinetics between East Asians and Caucasians and the role of genetic polymorphisms. J Clin Pharmacol 2004; 44: 1083-1105.

28. Liao JK. Safety and efficacy of statins in Asians. Am J Cardiol 2007; 99: 410-414.

29. Rauchhaus M, Coats AJ, Anker SD. The endotoxin-lipoprotein hypothesis. Lancet 2000; 356: 930-933.

30. Turunen M, Olsson J, Dallner G. Metabolism and function of coenzyme Q. Biochim Biophys Acta 2004; 1660: 171-199.

31. Littarru GP, Tiano L. Bioenergetic and antioxidant properties of coenzyme Q10: Recent developments. Mol Biotechnol 2007; 37: 31 37.

32. McMurray JJ, Dunselman P, Wedel H, Cleland JG, Lindberg M, Hjalmarson A, et al. Coenzyme Q10, rosuvastatin, and clinical outcomes in heart failure: A pre-specified substudy of CORONA (controlled rosuvastatin multinational study in heart failure). J Am Coll Cardiol 2010; 56: 1196-1204.

\section{Supplementary Files}

Supplementary File 1

Appendix S1. Independent Endpoint Committee

Appendix S2. Independent Data and Safety Monitoring Board

Appendix S3. Steering Committee

Appendix S4. Investigators

Please find supplementary file(s);

http://dx.doi.org/10.1253/circj.CJ-12-1062 